\title{
Abdominal Tuberculosis
}

\author{
Narayanappa $\mathrm{D}^{1}$, Thirumala $\mathrm{CR}^{2}$
}

\begin{abstract}
Abdominal tuberculosis is a common form of extrapulmonary tuberculosis constituting $11 \%$ of the cases; lleocaecal region is the most commonly affected area followed by ascending colon, jejunum, appendix, duodenum, stomach, esophagus, sigmoid colon, and rectum. Diagnosis is a challenge which involves basic work up like computed tomography (CT) scan, esophageal ultrasonography (USG), ascitic fluid adenosine deaminase (ADA) and special investigations like capsule endoscopy, balloon enteroscopy, TB-PCR, GeneXpert, diagnostic laparoscopy are being increasingly used. Standard antitubercular drugs are the first line of treatment, and they are usually highly effective for intestinal TB. Sixmonths therapy is as effective as 9 months of therapy. Surgery is usually reserved for patients who have developed complications or obstruction not responding to medical management. Multidrug resistance (MDR) has been observed in $13 \%$ of MTB isolates in abdominal tuberculosis.
\end{abstract}

Keywords: Abdominal tuberculosis, Extrapulmonary tuberculosis, Multidrug resistance tuberculosis, RNTCP, Tubercular peritonitis.

Pediatric Infectious Disease (2019): 10.5005/jp-journals-10081-1105

\section{INTRODUCTION}

$\mathrm{T}$ uberculosis is a major public health problem in India, with more than 1 million tuberculosis cases reported in children. As per the World Health Organization (WHO) global TB report of 2018, India tops the list of countries with reported tuberculosis cases. Abdominal tuberculosis is the most common type of extra-pulmonary tuberculosis. The exact incidence of abdominal TB is not known. Abdominal TB comprises of tuberculosis of gastrointestinal tract, peritoneum, omentum, mesentery, and its lymph nodes and other abdominal organs such as liver, spleen, and pancreas. We need to have a high index of suspicion to diagnose abdominal TB as it mimics other common abdominal diseases. Failure to suspect and diagnose abdominal TB can lead to high morbidity and mortality. ${ }^{1}$

\section{Pathophysiology}

Primary abdominal tuberculosis is when infection occurs through ingestion of milk or food infected with Mycobacterium bovis. Primary abdominal TB is rare nowadays. ${ }^{2}$

Secondary abdominal TB is due to tubercular focus elsewhere in the body, and it is acquired in the following ways:

- Dissemination of primary pulmonary tuberculosis

- Swallowing of infected sputum

- Hematogenous dissemination

- Spread from infected adjacent organs like fallopian tubes

- Lymphatic spread from infected mesenteric lymph nodes

\section{Predisposing Factors for Intestinal Tuberculosis ${ }^{3}$}

- Rich in lymphoid tissue: Peyer's patches and lymph nodes

- AFB affinity for lymphoid tissue

- Number of bacilli ingested

- Virulence of bacilli

- Nutritional and immunological status

- Alkaline $\mathrm{pH}$ in the small and large intestine ${ }^{4}$

- $\quad$ Stasis in the ileocecal area (lleal break)

- More of water absorption and no digestive activity

\section{Sites of Involvement in Abdominal Tuberculosis ${ }^{5}$}

Intestines are the most common site of abdominal TB, where involvement of ileum is seen in $43.6 \%$ of cases. Peritoneum, colon,
${ }^{1}$ Professor, ${ }^{2}$ Senior Resident

1,2Department of Pediatrics, JSS Medical College, JSS AHER, Mysuru, Karnataka, India

Corresponding Author: Narayanappa D, Professor, Department of Pediatrics, JSS Medical College, JSS AHER, Mysuru, Karnataka, India, e-mail: sinchabhi@yahoo.com

How to cite this article: Narayanappa D, Thirumala CR. Abdominal Tuberculosis. Pediatr Inf Dis 2019;1(1):19-22.

Source of support: Nil

Conflict of interest: None

and rectum are other frequently involved sites. In younger children, peritoneum and mesenteric nodes are most commonly involved.

\section{Pathology 6}

Based on the pathology, abdominal TB is classified as shown in Table 1.

Ulcerative form: Ulcers are usually transverse to the long axis of the bowel called Girdle ulcers. Areas of normal mucosa may be found amidst diseased segments and are called as skip lesions. Depth of the ulcers varies from submucosa to muscularis propria or even serosa. Healing and fibrosis lead to napkin ring strictures causing obstructive symptoms.

Hypertrophic form: In contrast to the ulcerative form, stricturous hypertrophic form may occur in young well-nourished patients.Cecum is the commonest site affected. Usually, the clinical setting is of low volume infection by less virulent organisms in a relatively healthy host.

\section{Presentation According to Site of Involvement ${ }^{7}$}

Abdominal tuberculosis is a diagnostic challenge as it presents with nonspecific symptoms and has varied manifestations. The clinical presentation can be acute, chronic or acute on chronic.

\section{Frequency of Presentation of Signs and Symptoms ${ }^{8}$}

Pain abdomen and weight loss being the most common clinical presentation followed by anorexia, fever, chronic diarrhea, and vomiting. Most important clinical signs being abdominal distension, followed by doughy abdomen and hepato-splenomegaly. Less commonly visible peristalisis and ascites can be demonstrated.

(C) The Author(s). 2019 Open Access This article is distributed under the terms of the Creative Commons Attribution 4.0 International License (https://creativecommons. org/licenses/by-nc/4.0/), which permits unrestricted use, distribution, and non-commercial reproduction in any medium, provided you give appropriate credit to the original author(s) and the source, provide a link to the Creative Commons license, and indicate if changes were made. The Creative Commons Public Domain Dedication waiver (http://creativecommons.org/publicdomain/zero/1.0/) applies to the data made available in this article, unless otherwise stated. 
Table 1: Abdominal tuberculosis

\begin{tabular}{lllll}
\hline Intestinal & Peritoneal & Omental & Military & TB of lymph nodes \\
\hline Ulcerative & Peritonitis & Rolled up & $\begin{array}{l}\text { Granular peritoneal } \\
\text { surface }\end{array}$ & Tabesmesenterica \\
Hypertrophic & $\begin{array}{l}\text { Ascitic: } \\
\text { (a) Generalised } \\
\text { (b) Localised }\end{array}$ & Miliary TB & & Retroperitoneal \\
Ulcerohypertrophic & $\begin{array}{l}\text { Dry plastic type } \\
\text { Fibroplastic }\end{array}$ & & Peripancreatic \\
\hline
\end{tabular}

\section{Peritoneal Tuberculosis}

Tuberculous peritonitis is more common among females than males. The risk factors for peritoneal TB are HIV infection, liver cirrhosis, diabetes mellitus, malignancy, and continuous ambulatory peritoneal dialysis. It is manifested as abdominal distention, ascites and cystic lump secondary to loculated ascites.

Constitutional symptoms of fever and night sweats may be present. Esophagus: Esophagus is rarely involved, mainly by extension of disease from adjacent lymph nodes in the mediastinum. Mid and upper esophagus are common sites. Low-grade fever, dysphagia, odynophagia are the presenting symptoms.

Gastric: Due to paucity of lymphoid tissue and the presence of an acid, the stomach is an uncommon site for intestinal TB. The ulcerative form is the most common form, and in $80 \%$ of the cases, ulcers are seen on the lesser curvature of the stomach. Symptoms are nonspecific and include abdominal pain, nausea, vomiting, GI bleed, fever, and weight loss.

Duodenal: This is also a rare site of tuberculosis. Symptoms include nonspecific dyspeptic symptoms, fever and weight loss.

Colorectal: It can present with a nonspecific symptom or with weight loss, anemia and lower GI bleed. There can be diffuse or segmental colitis.

Anal tuberculosis: Anal tuberculosis is also uncommon and presents as anal discharge, perianal swelling, pain, and multiple fistula.

Hepatobiliary tuberculosis: Presentation can be in the form of pain (usually localized to right hypochondrium), fever, jaundice, hepatomegaly, tenderness, splenomegaly, anemia or ascites.

Splenic tuberculosis: Spleen usually gets enlarged as a part of disseminated tuberculosis. Occasionally there can be massive splenomegaly also.

\section{DiAgNOSTIC APPROACH ${ }^{9}$}

- Diagnosis of ATB needs strong clinical suspicion and needs to fulfill the following criteria

- Demonstration of an acid-fast bacillus (AFB) in the lesion or ascitic fluid. Growth of $M$. tuberculosis on the culture of tissue or ascitic fluid.

- Histological evidence of caseating granuloma

- Operative evidence of ATB

- Good therapeutic response to chemotherapy.

Granulomas have been reported in only $40 \%$ of ATB patients and may be present only in lymph nodes. They are absent in enteric lesions. In a host with good defense response or low virulence of the organism noncaseating granulomas may be seen. Caseation is a histological marker for ATB and helps in differentiating it from Crohn's disease. ATB is a paucibacillary disease; therefore, the working diagnosis is mainly based on history, clinical findings, and histology. Most of the times the demonstration of AFB in tissue or culture is not possible in children.

\section{Definitive Diagnosis Techniques}

Demonstration of AFB by:

- Fine needle aspiration cytology (FNAC): FNAC from intraabdominal mass due to lymph nodes, rolled up omentum, and hypertrophied lesion of the intestine. Aspiration can be guided by ultrasound or CT if a mass is deep-seated .

- Ascitic fluid for AFB and culture: The yield of organisms from ascitic fluid either by AFB staining or by culture is very low. AFB can be demonstrated from the ascitic fluid in $<3 \%$ cases, and tubercle bacilli can be cultured in $<20 \%$ cases.

- AFB in the biopsy tissue: Demonstration of AFB in the biopsy material obtained by endoscopy confirms the diagnosis. M. tuberculosis can also be cultured from biopsied tissue/ material or the specimen obtained and can be subjected to PCR for detection. Various studies have reported the sensitivity of endoscopic biopsy from $30 \%$ to $80 \% .^{10}$

\section{Supportive Diagnosis Methods ${ }^{11}$}

- Mantoux test: It has low sensitivity and specificity, its positivity alone is not reliable for diagnosis. Negative Mantoux test does not exclude tuberculosis.

- X-ray chest: X-ray chest incidence of pulmonary tuberculosis varies from $19 \%$ to $58 \%$ in abdominal tuberculosis. Abnormal chest X-ray is seen in $50 \%$ to $75 \%$ cases of tuberculous peritonitis.

Family screening: A definite history of contact is not always present but various case series reported had a positive family history in $37 \%$ to $66 \%$ cases of ATB. X-ray chest of all family members and Mantoux test of preschool age children should be done when there is a history of contact.

- Barium studies: Barium meal follow through (BMFT) is considered as best in demonstrating bowel lesions, their extent, and site in up to $74 \%$ of cases. It shows ulcerations, fistulae, multiple strictures with segmental dilatation of bowel loops and fixity and matting of bowel loops, accelerated intestinal transit, hypersegmentation of the barium column (chicken intestine), precipitation, flocculation and dilution of the contrast material, stiffened and thickened folds; luminal stenosis with smooth but stiff contours (hourglass stenosis).

- Barium enema features suggestive of abdominal TB: Fleischner or inverted umbrella sign is characteristic.

* Conical cecum: Cecum is shrunken in size and pulled out of the iliac fossa due to contraction and fibrosis of the mesocolon.

* Gooseneck deformity: Loss of normal ileocecal angle and dilated terminal ileum, appearing suspended from a retracted, fibrozed cecum.

* Purse string stenosis: Localized stenosis opposite the ileocecal valve with a rounded off smooth cecum and a dilated terminal ileum.

* String sign: There is a persistent narrow stream of barium indicating stenosis. 
- Percutaneous fistulogram: Contrast can be injected through the fistulous tract to delineate the enteric communication. ${ }^{12}$

- Abdominal ultrasound: Early ATB can be suspected when the mesenteric thickness is more than $15 \mathrm{~mm}$, increased mesenteric echogenicity due to fat deposition and lymphatic obstruction and mesenteric lymphadenopathy.

- Intra-abdominal fluid can be seen which may be free or loculated. USG is superior to CT in detecting ascites.

- Club sandwich or sliced bread sign-localized fluid between radially oriented bowel loops due to local exudation from the inflamed bowel forms. ${ }^{13}$

- Ascitic fluid analysis: The peritoneal fluid is exudative in nature, which is either straw-colored or clear. The fluid analysis shows proteins more than $3 \mathrm{~g} / \mathrm{dL}$, cells more than 1000/cumm (mostly lymphocytes), ascitic/ blood glucose ratio less than 0.96 and serum albumin ascitic gradient (SAAG) level less than $1.1 \mathrm{~g} /$ dL. ADA in the ascitic fluid has been considered to be a useful screening test in children with ATB. A level of more than 33U/L has a sensitivity and specificity of $93 \%$ and $96 \%$ respectively with a positive predictive value of $93 \%$.

- Malabsorption studies: Abnormal malabsorption tests seen in these children are not diagnostic. Abnormal parameters were seen in $30.8 \%$ of cases in pediatric series.

\section{Newer Modalities ${ }^{14}$}

- Virtual CT enteroclysis and virtual CT colonoscopy

- Sonoenteroclysis

- Serodiagnosis

* Monoclonal antibodies: Monoclonal antibodies have been developed against $M$. tuberculosis. Monoclonal antibodies (IgG type) also called TB 72, are raised in 74\% of pulmonary tuberculosis.

* Antigens: To detect the presence of mycobacterial antigen in the blood, very sensitive methods like RIA and ELISA have been used. ELISA is highly sensitive for the detection of antigen in CSF.

* Polymerase chain reaction (PCR): It has low sensitivity and specificity in abdominal TB, unlike pulmonary tuberculosis. It is a rapid diagnostic test which identifies bacterial DNA, most commonly used target is insertion sequence IS6110 which is present in 60\% of specimens.

* Interferon-gamma release assays (IGRA): IFN-gamma responses to M. tuberculosis-specific antigens (ESAT-6, CFP-10, TB 7.7) are being used in the in vitro diagnostic tests.

* Chemokine IP-10 (CXCL 10) has been introduced as a specific diagnostic marker for infection with $M$. tuberculosis. According to recent RNTCP/IAP paediatric /TB guidelines 2019 newer diagnostic modalitie like CBNAAT,Line probe assay, MGIT are recommended. ${ }^{15-18}$

* Differential diagnosis: Crohn's disease has similar features, and differentiating point are modularity and aphthous ulcers, granulomas are noncaseating and compact. Whereas in tuberculosis, hypertrophic lesions, pseudopolyps, and caseating granulomas are seen. Fever and circumferential deep linear ulcers are seen with intestinal TB whereas transverse patulous ulcers are seen in Crohn's disease.

The differential diagnosis for caseating granulomas being fungal infections like histoplasmosis and Cryptococcus.

Another differential diagnosis mainly presenting with ascites are lymphomas, end-stage liver diseases, and spontaneous bacterial peritonitis.

\section{Complications of Abdominal TB ${ }^{16}$}

Obstruction,stricture,percutaneous fistula, enteric fistula due to intestinal hemorrhage.

Abdominal cocoon', also known as 'sclerosing encapsulating peritonitis' (SEP), which is a rare condition that is characterized by the encasement of the small bowel by a fibro collagenic cocoon-like sac that causes intestinal obstruction.

\section{Treatment $^{19}$}

Antituberculous chemotherapy is the mainstay in the management of abdominal tuberculosis. Surgical options are reserved for tissue diagnosis or treatment of complications. Chemotherapy consists of 4 drugs and for 6 months. ${ }^{18}$ Short-course chemotherapy is well studied in adults. Even though initial responses are good, it is associated with frequent relapses. So many physicians tend to extend therapy up to 12 months. Short-course chemotherapy and the role of pyrazinamide have not been evaluated in children with abdominal tuberculosis. There is a report of short-term chemotherapy in adults with a threedrug regimen of $\mathrm{INH}$, rifampicin, and pyrazinamide for 2 months followed by INH and rifampicin for 4 months. A favorable response was seen in $97 \%$ in short-course therapy versus $92 \%$ in the standard treatment group. However, toxicity was $26 \%$ in short-course group and $13 \%$ in the standard therapy group.

Short-course chemotherapy regimen consists of INH (4-6 mg/ $\mathrm{kg}$ per day), rifampicin ( $10 \mathrm{mg} / \mathrm{kg} /$ day), ethambutol ( $15 \mathrm{mg} / \mathrm{kg} /$ day) and pyrazinamide ( $25 \mathrm{mg} / \mathrm{kg} /$ day) for initial 2 months. ${ }^{23}$ After 2 months ethambutol and pyrazinamide are stopped, but INH and rifampicin in the same dose are continued for 7 months. If the nodal tuberculosis is dominating then antituberculosis therapy (ATT) is continued for 9-12 months. In ATB nodal tuberculosis is always there; therefore consensus is to continue ATT for 9 months to avoid relapse. If $M$. bovis is isolated pyrazinamide can be discontinued because of its innate resistance to the drug. But it usually takes 2 to 3 months to make this differentiation. Ninety percent of patients with enteric tuberculosis and peritoneal tuberculosis will respond to medical therapy alone if treatment is started earlier.

Hepatic transaminases are monitored periodically till completion of therapy. When hepatotoxicity is present, both the

Criteria for Diagnosis of Abdominal Tuberculosis ${ }^{15}$

\begin{tabular}{|c|c|}
\hline Definitive & Modified \\
\hline - Histological evidence of tubercles with caseating necrosis & - Histological evidence of caseating granulomas or AFB \\
\hline $\begin{array}{l}\text { A good typical gross description of operative findings with } \\
\text { biopsy of mesenteric nodes showing histological evidence of } \\
\text { tuberculosis }\end{array}$ & - Presence of M. tuberculosis in sputum or tissue or ascitic fluid \\
\hline $\begin{array}{l}\text { - Animal inoculation or culture of suspected tissue resulting in } \\
\text { the growth of } M \text {. tuberculosis }\end{array}$ & $\begin{array}{l}\text { - Clinical/radiological/operative evidence of proven tuberculosis } \\
\text { elsewhere with good therapeutic response }\end{array}$ \\
\hline - Histological demonstration of acid-fast bacilli in a lesion. & - Good therapeutic response to antitubercular drugs \\
\hline
\end{tabular}


drugs INH and rifampicin are stopped and alternate therapy is given till the time there is the recovery of hepatic injury. ${ }^{18}$

The role of corticosteroids in the management of ATB to decrease the formation of adhesions and stricture is not proven.

In enteric tuberculosis which is commonly associated with complications,surgery may be required. Antituberculosis therapy should be given preferably for 6-8 weeks before surgery and continued for at least 6-9 months after surgery.

\section{Role of Surgery}

The indications for surgical treatment are:

- Diagnostic

- Minilaparotomy and biopsy

- Laparoscopic biopsy in ascitic type

- Extra-abdominal lymph node biopsy. Exploratory laparotomy in the acute abdomen.

Management of complications like strictures, adhesive obstruction, fistulae or bleeding.

\section{Outcomes}

Mortality being $8-50 \%$ and common among those with delay in initiating treatment and with underlying cirrhosis.

\section{References}

1. Abughali N, V anderKuyp F, Annable W, et al. Congenital tuberculosis. PediatrInfecDis J 1994;13:773-791.

2. MMWR Mor Mort WkI Report. Congenital pulmonary tuberculosis associated withmaternal cerebral tuberculosis-2005;54:1062-1066.

3. Chotpitaysunondh T, sangtawesin V. Congenital tuberculosis. J Med Assoc Thai 2003;86suppl:S689-695.

4. Diar H, Velaphi $\mathrm{S}$. Congenital tuberculosis as a proxy to maternal tuberculosis: a case report. J Perinatol 2009;29:709-711.

5. Das A, Arora J, Rana T, et al. Congenital tuberculosis: The value of laboratory investi-gations in diagnosis. AnnTropPaediatr 2008;28:137-141.
6. Bhat RY, Rao A, Althaf, et al. An evolved diagnosis of congenital tuberculosis in avery low birth weight premature neonate. Int J Tuberc Lung Dis 2008;12:344-345.

7. Doudier B, Mosnier E, Rovery C, et al. Congenital tuberculosis after in vitro fertilization. Pediatr Infect Dis J 2008;27:277-278.

8. Al-Katawee YA, Al-Mahmood LA, Al-Showaier AS. Congenital tuberculosis presenting as cutaneous disease in a premature infant. Saudi Med J 2007;28:1739-1740.

9. Lee LH, Levea CM, Graman PS. Congenital tuberculosis in a neonatal intensive care unit: Case report, epidemiological investigation, and management of exposures. Clin Infec Dis 1999;29:467.

10. Hageman JR. Congenital and perinataltuberculosis:Discussion of the difficult issues of diagnosis and management. J Perinatol 1998;18: 389-394.

11. Starke JR. Tuberculosis. An old disease but a new threat to the mother fetus and neonate.Clin Perinatol 1997;24:10727.

12. Balaka B, Bakande B, Douti $K$, et al. Tuberculosis in the newborn: Recrudescence in areas with high endemic HIV infection. Med Trop (mars) 2004;64:367-371.

13. Naranbai RC. Congenital tuberculosis localised to the ear. Arch Dis Child 1989;64:738-740.

14. Scnbil N, Sahin F, Caglar MK, et al. Congenital tberculosis of the ear and parotid gland. Pediatr Infect Dis 1997;16:1090-1091.

15. Hageman J, Shulman S, Schreiber M, et al. Congenital tuberculosis: Critical reappraisal of clinical findings and diagnostic procedures. Pediatrics 1980;66:980-984.

16. Treatment of tuberculosis and tuberculosis infection in adults and children: Official statement of the American Thoracic Society. Am J Respir Crit Care Med 1994;149:1359-1375.

17. Neyaz Z, Gadodia A, Gamanagatti S, et al. Imaging findings of congenital tuberculosis in three infants. Singapore Med J 2008;49: e42-e46.

18. Draft updated IAP RNTCP Ped TB guidelines 2019, central TB division MOHFW New Delhi India;21

19. Miller FJW. Tuberculosis in children.Evaluation, Epidemiology, Treatment, Prevention.(1st edn). New Delhi, Churchill Livingstone 1982;220-224. 